\title{
THE UNIVERSITY of EDINBURGH
}

\author{
Edinburgh Research Explorer
}

\section{Photo-ionization and fragmentation of Sc3N@C80 following excitation above the Sc K-edge}

Citation for published version:

Obaid, R, Schnorr, K, Wolf, TJA, Takanashi, T, Kling, NG, Kooser, K, Nagaya, K, Wada, S, Fang, L, Augustin, S, You, D, Campbell, EEB, Fukuzawa, H, Schulz, CP, Uleda, K, Lablanquie, P, Pfeifer, T, Kukk, E \& Berrah, N 2019, 'Photo-ionization and fragmentation of Sc3N@C80 following excitation above the Sc Kedge', The Journal of Chemical Physics, vol. 151, no. 10, pp. 104308. https://doi.org/10.1063/1.5110297

\section{Digital Object Identifier (DOI):}

10.1063/1.5110297

Link:

Link to publication record in Edinburgh Research Explorer

Document Version:

Peer reviewed version

Published In:

The Journal of Chemical Physics

\section{General rights}

Copyright for the publications made accessible via the Edinburgh Research Explorer is retained by the author(s) and / or other copyright owners and it is a condition of accessing these publications that users recognise and abide by the legal requirements associated with these rights.

\section{Take down policy}

The University of Edinburgh has made every reasonable effort to ensure that Edinburgh Research Explorer content complies with UK legislation. If you believe that the public display of this file breaches copyright please contact openaccess@ed.ac.uk providing details, and we will remove access to the work immediately and investigate your claim. 
2 K-edge

Razib Obaid, ${ }^{1, \text { a) }}$ Kirsten Schnorr, ${ }^{2}$ Thomas J. A. Wolf, ${ }^{3}$ Tsukasa Takanashi, ${ }^{4}$ Nora G. Kling, ${ }^{1}$ Kuno Kooser, ${ }^{5,6}$ Kiyonobu Nagaya, ${ }^{7,8}$ Shin-ichi Wada, ${ }^{9}$ Li Fang, ${ }^{10}$ Sven Augustin, ${ }^{2}$ Daehyun You, ${ }^{4}$ Eleanor E. B. Campbell, ${ }^{11}$ Hironobu Fukuzawa, ${ }^{4,8}$ Claus-Peter Schulz, ${ }^{12}$ Kiyoshi Ueda, ${ }^{4,8}$ Pascal Lablanquie, ${ }^{13}$ Thomas Pfeifer, ${ }^{2}$ Edwin Kukk, ${ }^{5}$ and Nora Berrah ${ }^{1}$

1) Department of Physics, University of Connecticut, USA

2)Max-Planck-Institut für Kernphysik, Germany

3) PULSE Institute, SLAC National Accelerator Laboratory, USA

${ }^{4)}$ Institute of Multidisciplinary Research for Advanced Materials, Tohoku University, Japan

${ }^{5)}$ Deparment of Physics, University of Turku, Finland

${ }^{6)}$ Institute of Physics, University of Tartu, Estonia

${ }^{7)}$ Department of Physics, Kyoto University, Japan

${ }^{8)}$ RIKEN SPring-8 Center, Japan

9) Department of Physical Science, Hiroshima University, Japan

${ }^{10)}$ Department of Physics, The Ohio State University, USA

11) EastCHEM and School of Chemistry, University of Edinburgh,

United Kingdom

${ }^{12)}$ Max-Born-Institut, Germany

${ }^{13)}$ Laboratoire de Chimie Physique-Matière et Rayonnement, Sorbonne Université, France 
We have investigated the ionization and fragmentation of a metallo-endohedral fullerene, $\mathrm{Sc}_{3} \mathrm{~N} @ \mathrm{C}_{80}$ using ultrashort (10 fs) x-ray pulses. Following selective ionization of a Sc $(1 s)$ electron $(h v=4.55 \mathrm{keV})$, an Auger cascade leads predominantly to either a vibrationally cold multiply charged parent molecule or multi-fragmentation of the carbon cage following a phase transition. In contrast to previous studies, no intermediate regime of $\mathrm{C}_{2}$ evaporation from the carbon cage is observed. A time-delayed, hard x-ray pulse $(h v=$ $5.0 \mathrm{keV}$ ) was used to attempt to probe the electron transfer dynamics between the encapsulated Sc species and the carbon cage. A small but significant change in the intensity of Sc-containing fragment ions and coincidence counts for a delay of 100 fs compared to 0 fs, as well as an increase in the yield of small carbon fragment ions, may be indicative of incomplete charge transfer from the carbon cage on the sub-100 fs timescale.

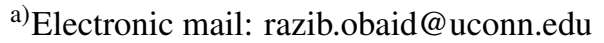


26 Endohedral (or internally doped) fullerenes, are intriguing systems that bridge the gap between ${ }_{27}$ molecular and nano-systems ${ }^{1,2}$. Like $\mathrm{C}_{60}$, there is much to learn about their behavior when they 28 are excited with photons, in particular in the hard X-ray regime. Endohedral fullerenes are of great ${ }_{29}$ interest to study due to their unique properties, including electron transfer between the encaged 30 species and the carbon cage ${ }^{2}$, and their potential use in molecular electronics and organic photo31 voltaics. The understanding derived from the photoionization of carbon nanomaterials can provide 32 insight towards optimizing their properties for use in these applications ${ }^{3}$. Endohedral fullerenes 33 have been studied with optical lasers ${ }^{4,5}$ and synchrotron radiation ${ }^{6-9}$. Synchrotron studies, which 34 allow for core-level ionization of the encapsulated species, have indicated that there is a much 35 higher fragmentation propensity if the inner species becomes highly excited ${ }^{7}$, especially compared 36 to the level of fragmentation observed following excitation/ionization of valence electrons by op${ }_{37}$ tical lasers ${ }^{5}$. The most intriguing aspect of the endohedral systems compared to empty fullerenes ${ }_{38}$ is the mutual influence of the electrons from the cage and the electrons from the encapsulated 39 moiety, which may significantly modify the dynamics ${ }^{10}$.

40 Like synchrotron radiation, x-ray free electron lasers (FEL) can access the core levels of the ${ }_{41}$ inner moiety of the endohedral fullerene ${ }^{11}$. The brilliance and photon energy tunability of the ${ }_{42}$ FEL allows for site-specific photon absorption, and because they have short pulse durations, also 43 allows, in principle, for following the dynamics of the molecule ${ }^{11,12}$. Most of the early experiments ${ }_{44}$ involving FELs have been on understanding the nature of the interaction of light atoms, such as ${ }_{45}$ neon $^{13}$, and small molecules, such as $\mathrm{N}_{2}{ }^{14,15}$. Recently, polyatomic molecules ${ }^{12}$ and fullerenes ${ }^{16}$ ${ }_{46}$ have been studied, with an emphasis on understanding the ionization dynamics involved when 47 multiple x-rays are absorbed causing ionization and subsequent Auger decay, which contribute 48 substantially to what is known as "radiation damage" 17.

49 The aim of the present experiment was to investigate $\mathrm{Sc}_{3} \mathrm{~N} @ \mathrm{C}_{80}$ by site-selectively ionizing ${ }_{50}$ the Sc $(1 s)$ with an approximately 10 fs duration hard x-ray pulse at $4.55 \mathrm{keV}$ photon energy in51 ducing core-ionization followed by an Auger cascade. This investigation allows us to address how 52 electronic rearrangement affects (i) the nuclear motion leading to atomic rearrangement, (ii) bond ${ }_{53}$ elongation and breaking, (iii) fragmentation of the moiety (inner species) and the cage, and (iv) ${ }_{54}$ bond (re)-forming. For Sc, it is known that for a K-shell vacancy, the radiative to non-radiative 55 branching ratio is 0.18:0.82, while for the L-shell vacancy, the radiative yield is negligible com- 
Publisłaijagged to non-radiative relaxation which primarily occurs via Coster-Kronig type decay through ${ }_{57}$ the LMM process ${ }^{18}$. Thus, following removal of a K-shell electron of Sc, further ionization of ${ }_{58}$ the whole system is expected to proceed via Auger cascade or impact ionization of the cage. It is ${ }_{59}$ expected from the binding energy of the electrons in Sc, that KLL $\left(2 s^{-2} / 2 s^{-1} 2 p^{-1} / 2 p^{-2}\right)$ Auger ${ }_{60}$ decay will produce a free electron having a kinetic energy (KE) of about $3.5-4.0 \mathrm{keV}$. Likewise, ${ }_{61} \mathrm{LMM}\left(3 s^{-1} 3 p^{-1} / 3 p^{-2}\right)$ decay creates $\sim 330-400 \mathrm{eV}$ electrons ${ }^{19}$. The high energy electrons are ${ }_{62}$ expected to escape the cage with a low probability for further excitation of the molecule, while the ${ }_{63}$ few-hundred $\mathrm{eV}$ electrons are expected to have a higher probability to undergo inelastic collisions 64 with the cage ${ }^{20,21}$. A cartoon schematic of this mechanism is shown in Fig. 1 (a). We aimed to ${ }_{65}$ study transient structural changes by using a delayed second x-ray probe pulse, at a photon energy 66 of $5.0 \mathrm{keV}$, through monitoring the production of fragment ions.

\section{${ }_{67}$ II. EXPERIMENT}

The experiment was carried out at beamline BL3, EH4c hutch ${ }^{22}$ of the SPring-8 Angstrom ${ }_{69}$ Compact free electron LAser (SACLA) ${ }^{23}$ using a time-of-flight ion spectrometer ${ }^{24}$. Both the 70 "pump" and the "probe" pulses were produced by the same electron bunch using a variable length ${ }_{71}$ undulator scheme ${ }^{25}$. For this experiment, a pump pulse with $4.55 \mathrm{keV}$ photon energy $(\mathrm{FWHM}=$ $7213.11 \mathrm{eV})$ and a probe pulse with $5.0 \mathrm{keV}$ photon energy $(\mathrm{FWHM}=69.79 \mathrm{eV})$ were used. Since 73 the Sc atoms have an average charge state of $2.4+$ in the endohedral complex, the pump pulse was ${ }_{74}$ tuned to be well above the K-edge of Sc $\left(4492.8 \mathrm{eV}^{26}\right)$, so that $\mathrm{Sc}^{3+}$ could be ionized by single 75 photon absorption. Meanwhile, the probe pulse was chosen to be about $5.0 \mathrm{keV}$ for two reasons: 76 1) the photon energy of $5.0 \mathrm{keV}$ sufficiently allows for ionization to highly charge states, above ${ }_{77} 4+$, and 2) the difference in photon energies between the two pulses needed to be large enough 78 such that the pump and the probe could be monitored independently by an in-line spectrometer ${ }^{27}$. ${ }_{79}$ Both beams were focused by a Kirkpatrick-Baez (KB) mirror system to a focal spot size of about ${ }_{80} 1.7 \times 1.8 \mu^{2}$ (FWHM). Just prior to the experiment, the beam sizes were individually measured 81 by using an edge scanning method that utilizes a $200 \mu \mathrm{m}$ gold wire to create an intensity profile ${ }^{28}$. ${ }_{82}$ During the experiment, the spatial overlap of the pump and the probe was checked for the two ${ }_{83}$ delay points by using another $200 \mu \mathrm{m}$ gold wire at the interaction region, since the scheme ${ }^{25}$ used ${ }_{84}$ for the production of the pump and the probe pulses did not guarantee automatic spatial overlap ${ }_{85}$ following changes in the undulator gap of the electron bunch. The repetition rate of the pulses was 


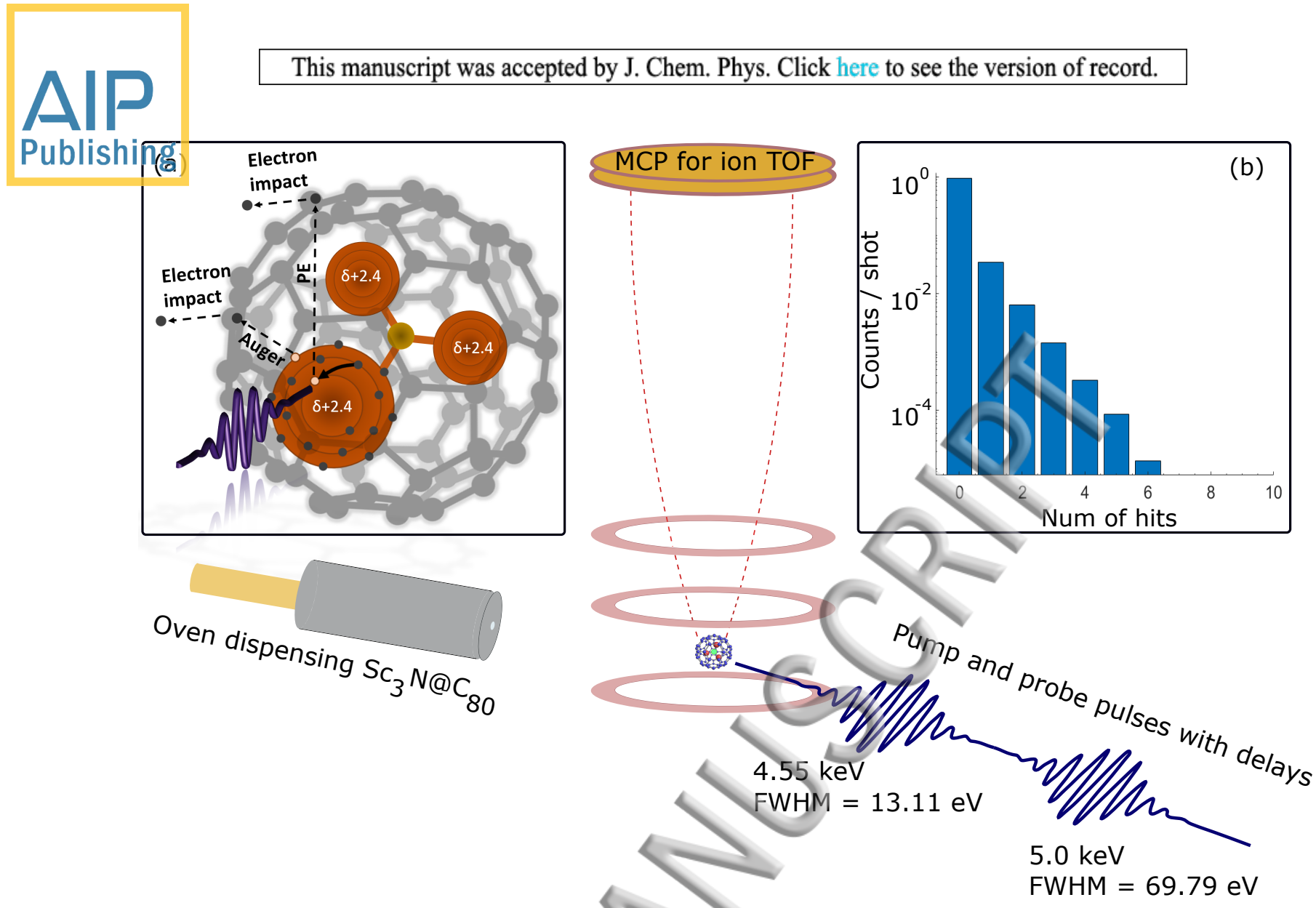

FIG. 1. Schematic of the experiment. An oven is used to vaporize the $\mathrm{Sc}_{3} \mathrm{~N} @ \mathrm{C}_{80}$ sample, which is directed to the interaction region though a nozzle. The low-density molecular beam is crossed by the focused FEL pulses in the center of a time-of-flight spectrometer. A moderate extraction field applied to the spectrometer electrodes directs ions to an MCP detector. In inset (a), a cartoon of the electron impact ionization of the cage is shown following the removal of a $1 s$ electron (photoelectron, PE) from Sc by the x-ray photon. Inset (b) shows the number of ion hits on the MCP at 100 fs delay (note that a similar hit rate is also observed for 0 fs delay).

${ }_{86} 60 \mathrm{~Hz}$, and the duration of these two pulses were estimated to be about $10 \mathrm{fs}$ each, as measured us87 ing a spectrometer consisting of an analyzer flat crystal of silicon ${ }^{29}$ prior to the experiment. Since 88 these measurements require their own chamber, the pulse duration was measured ex situ before the ${ }_{89}$ beamtime. The temporal jitter between the two pulses was estimated to be a few fs due to the elec90 tron bunch spacing, as measured previously for this scheme ${ }^{25}$. Using an in-line spectrometer ${ }^{27}$, ${ }_{91}$ the shot-to-shot pulse energy was monitored to be about $105 \mu \mathrm{J}$ for the pump and $110 \mu \mathrm{J}$ for the ${ }_{92}$ probe, and the energy fluctuation between the two pulses were found to be about $20 \%$ of the mean ${ }_{93}$ for each arm, as also shown in Fig. S1. 
Publisłaing The experimental setup is shown schematically in Fig. 1. The $\mathrm{Sc}_{3} \mathrm{~N} @ \mathrm{C}_{80}$ sample (97\% pu${ }_{95}$ rity) was procured from SES Research. The sample was converted to an effusive vapor by using 96 a sample dispenser oven ${ }^{16}$ which evaporated $\mathrm{Sc}_{3} \mathrm{~N} @ \mathrm{C}_{80}$ into its vapor phase at 970 - $1000 \mathrm{~K}$. ${ }_{97}$ The orientation of the oven was horizontal with respect to the spectrometer axis, and along the 98 polarization of the $\mathrm{x}$-ray pulses. We estimate the target density from the oven to be about $2 \times 10^{8}$ $99 \mathrm{~cm}^{-3}$.

100 The ions created were extracted by a uniform electric field of $550 \mathrm{~V} / \mathrm{cm}$ at the interaction 101 region, and were subsequently detected on a microchannel plate (MCP) detector. The ion hits on 102 the MCP were recorded using a digitizer and a software discriminator ${ }^{30}$. More details of the ion ${ }_{103}$ TOF spectrometer are described in detail elsewhere, see $^{24}$

${ }_{104}$ III. RESULTS AND DISCUSSION

105 At a fluence of about $40 \mu \mathrm{J} / \mu \mathrm{m}^{2}$ for each pulse (corresponding to an intensity of about $4 \times 10^{17}$ ${ }_{106} \mathrm{~W} / \mathrm{cm}^{2}$ ), the ionization mechanism is expected to be step-wise. First there is a photoionization ${ }_{107}(\mathrm{P})$ event followed by an intra-atomic Auger (A) decay until a stable charge state is reached. ${ }_{108}$ This mechanism was previously reported for FEL interactions with atoms, molecules, viruses and ${ }_{109}$ weakly bound clusters ${ }^{11-14,16,31-37}$. At the photon energies used in the experiments, the absorption 110 cross-section of the three scandium ions inside the cage is about $2.0 \times 10^{-19} \mathrm{~cm}^{2}(0.2 \mathrm{Mb})$ for the ${ }_{111} 4.55 \mathrm{keV}$ pump and $1.5 \times 10^{-19} \mathrm{~cm}^{2}(0.15 \mathrm{Mb})$ for the $5.0 \mathrm{keV}$ probe pulse. For the cage itself, 112 with 80 carbon atoms, the cross-section is about $3.0 \times 10^{-20} \mathrm{~cm}^{2}(0.03 \mathrm{Mb})$ for both of the pulses. ${ }_{113}$ The stability of the $\mathrm{Sc}_{3} \mathrm{~N} @ \mathrm{C}_{80}$ molecular complex depends on the oxidation state of the cage. It ${ }_{114}$ has been determined that electron sharing with the $\mathrm{Sc}_{3} \mathrm{~N}$ stabilizes the $\mathrm{C}_{80}$ cage, which attains the 115 stable icosahedral form by accepting about 6.3 electrons from the inner moiety ${ }^{38}$. This induces a ${ }_{116}$ partial charge of $2.4+$ on each Sc atom inside the cage, for the overall neutral $\mathrm{Sc}_{3} \mathrm{~N} @ \mathrm{C}_{80}$.

${ }_{117}$ Fig. 2 (a) shows the time-of-flight spectrum for $\mathrm{Sc}_{3} \mathrm{~N} @ \mathrm{C}_{80}$, for 0 fs delay between the two $118 \mathrm{X}$-ray pulses, with the different charge states of the parent molecular ions indicated. Multiple ${ }_{119}$ charge states of the parent molecule up to 4+ are observed, as seen previously for the case of the 120 single photon ionization of the Sc $2 p$ orbital $^{6}$ for the same target. The lack of singly-charged 121 parent molecules shows that the Sc $(1 s)$ photo-ionization plus Auger decay efficiently produces ${ }_{122}$ multiple charge states of $\mathrm{Sc}_{3} \mathrm{~N} @ \mathrm{C}_{80}$. It is striking that there is no evidence in the mass spectrum ${ }_{123}$ for the occurrence of $\mathrm{C}_{2}$ fragmentation from the carbon cage, i.e. $\mathrm{Sc}_{3} \mathrm{~N} @ \mathrm{C}_{78}, \mathrm{Sc}_{3} \mathrm{~N} @ \mathrm{C}_{76}$ and 

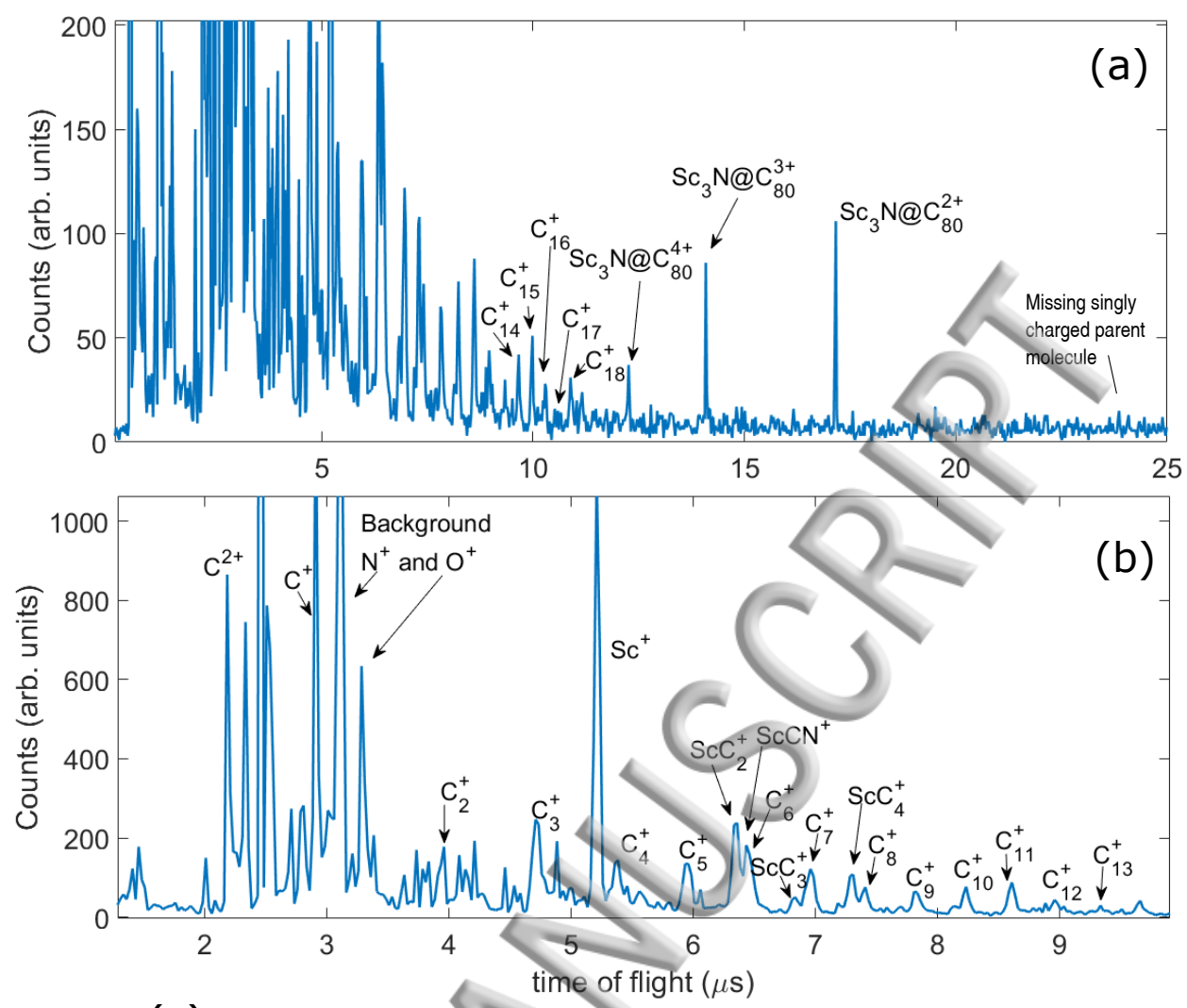

(c)

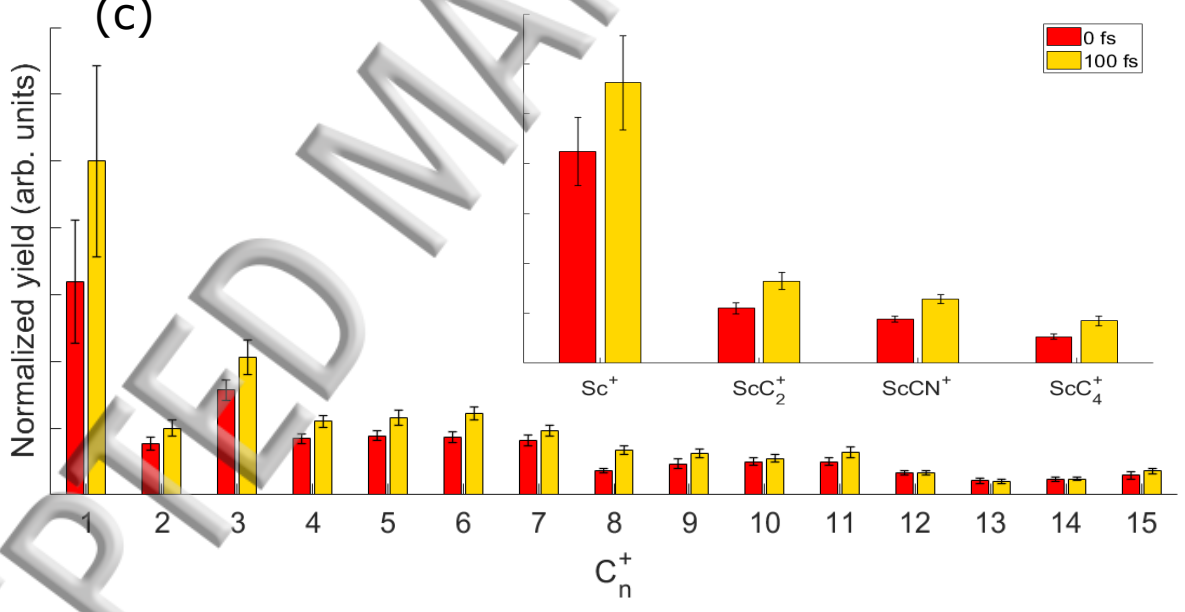

FIG. 2. Time-of-flight mass spectra for $\mathrm{Sc}_{3} \mathrm{~N} @ \mathrm{C}_{80}$ with 0 fs delay between the pump and probe pulses. (a) Overall spectra highlighting the different charge states of the parent molecule as well as different fragments. (b) Zoomed-in spectrum highlighting the smaller carbon cage fragment ions. (c) Yield, normalized by the number of FEL shots and pulse energies, comparing the production of carbon fragments for 0 and $100 \mathrm{fs}$ delays. The inset in (c) shows the normalized yield comparison for the Sc-containing fragments.

124 So on. Such typical "shrink-wrapping" behavior has been observed in previous studies where the ${ }_{125} \mathrm{Sc} 2 p$ orbital was photoionized ${ }^{6}$ and also in intense fs laser studies of $\mathrm{Ho}_{3} \mathrm{~N} @ \mathrm{C}_{80}{ }^{5}$. The lack 
Publisłaipfg $C_{2}$ fragmentation from the cage is a clear indication that the parent molecular ions visible in ${ }_{127}$ the mass spectrum have low internal energies. Note that the MCP detector was not floated in ${ }_{128}$ these experiments to provide high impact velocity, and thus the detection efficiency of the parent 129 molecular ions is expected to be significantly lower than that for the small carbon species in the ${ }_{130}$ experiments. The other striking observation is the lack of highly charged fragment ions. The ${ }_{131}$ highest fragment charge state that can be clearly distinguished in the spectra is $\mathrm{C}^{2+}$ with possibly ${ }_{132}$ a small amount of $\mathrm{Sc}^{2+}$. The low level of charging and corresponding lack of Coulomb explosion 133 is also manifested by the rather narrow ion peaks and the dominance of the detection of single 134 ions per shot (ca. $96 \%$ of all shots that yield an ion signal), as illustrated in Fig. 1 (b). The 135 most stable charge on the parent molecule is $2+$ and $3+$. This may be due to the stabilizing nature ${ }_{136}$ of the $\pi$ electron delocalization in the system following ionization, which corresponds to a larger ${ }_{137}$ gap between the HOMO and LUMO orbital for the resultant multiply charged parent molecule ${ }^{39}$. ${ }_{138}$ Fig. 2 (b) shows the time-of-flight spectrum for the various singly-charged carbon fragments. We 139 observe carbon fragments, $\mathrm{C}_{n}^{+}$for $\mathrm{n}<20$. Some doubly charged atomic carbon is also observed, 140 indicating that a portion of the carbon atoms in the cage lost multiple electrons either through ${ }_{141}$ their transfer from the cage to the moiety, or through electron collisions from the Auger electrons. ${ }_{142}$ What is surprisingly missing in the time-of-flight spectra is the observation of highly charged ${ }_{143}(>2+)$ states of Sc ions which one may have expected following Sc $(1 s)$ photo-ionization and ${ }_{144}$ subsequent Auger decay. That we mainly observe $\mathrm{Sc}^{+}$, and only a small amount of $\mathrm{Sc}^{2+}$, seems ${ }_{145}$ to be indicative of charge being efficiently transferred from the cage to the Sc ions.

${ }_{146}$ In Fig. 2 (c), we show the yield of the singly charged carbon fragments for the two delay points, 147 normalized to the number of shots and pulse energy per shot (see supplemental information figure ${ }_{148} \mathrm{~S} 1$ for the distribution of pulse energies for the two pump-probe experiments). The inset shows 149 the Sc-containing fragment ion yields, which includes $\mathrm{Sc}^{+}, \mathrm{ScC}_{2}^{+}, \mathrm{ScCN}^{+}$, and $\mathrm{ScC}_{4}^{+}$. The latter 150 three fragments are products requiring new bond formation since in the initial system, no direct 151 bond existed between the three $\mathrm{Sc}$ and the $\mathrm{C}_{80}$ cage. Such new bonds are typically observed fol152 lowing ionization and multi-fragmentation of the cage, as seen previously for the case of FEL and ${ }_{153}$ fs optical laser-induced fragmentation of $\mathrm{Ho}_{3} \mathrm{~N} @ \mathrm{C}_{80} 5,32$ as well as photo-ionization of Sc $(2 p)$ in ${ }_{154} \mathrm{Sc}_{3} \mathrm{~N} @ \mathrm{C}_{80}{ }^{6}$ and intense ns optical laser photoionization/fragmentation of $\mathrm{La} @ \mathrm{C}_{82}{ }^{4}$. On compar155 ison of the two sets of data, the normalized ion yield is slightly higher for the small carbon species 156 and, more significantly, the scandium-containing small fragments at 100 fs delay compared to 0 157 fs. Due to technical reasons of the two pulse scheme ${ }^{25}$, the only way single pulse spectra could be 
Publisłalingained is by filtering out the probe pulse using an Al filter. This resulted in some absorption of 159 the pump pulse, and thus the pulse energy for single pulse mode was different than what was used 160 for the two pulse experiment. Single pulse spectra obtained for pulse energies of $80 \mu \mathrm{J}$ and $520 \mu \mathrm{J}$ 161 show mostly similar trends in the relative intensities of the fragment ion peaks (see supplemental 162 information figures S2, S3, and S4). The pulse energy variation between the two delays (0 fs and $163100 \mathrm{fs}$ ) was about 5\%, which should not change the fact that what we are seeing is predominantly 164 single photon absorption (see supplemental information figure S5, which shows that the effect of 165 this pulse energy variation is negligible for multiple ionization- of parent molecules). Inside the 166 cage, each Sc has contributed about 3 electrons to the cage and the nitrogen atom. This implies ${ }_{167}$ that the $3 \mathrm{~d}$ and $4 \mathrm{~s}$ levels are not occupied. The x-ray absorption first leads to a KLL process, 168 leaving two L-shell holes. This can be followed by subsequent decay of two M-shell electrons 169 to fill the holes. Since direct double ionization has a much lower probability than single Auger 170 decay $^{40}$, this would leave the system with the 4 electrons removed from the Sc. Electrons from 171 the cage would then flow to the ionized Sc. If very little energy is transferred to the cage on the 172 way out or via the charge rearrangement process then one could expect to see up to $\mathrm{Sc}_{3} \mathrm{~N} @ \mathrm{C}_{80}^{4+}$. 173 If the system absorbed more than one photon, then we would expect to see higher charge states. ${ }_{174}$ However, the stability of endohedral fullerenes is also dependent upon the $\pi$-orbital delocaliza175 tion of the system ${ }^{39}$, and does not support high charge states beyond 5+. For the case of single 176 photon absorption, one can definitely create multiple charge states on the Sc site. However, as 177 also observed in previous studies of Sc $2 p$ ionization $^{6}$, competing processes of cascade ioniza178 tion and charge rearrangement from the cage to the moiety leads to fragmentation, as it becomes 179 more and more unfavorable for the system to remain stable with increasingly higher charge states. ${ }_{180}$ Therefore, further ionization of the cage would result in instability of the system, manifested by 181 cage opening/break up. This ensures that the products which will show time-dependent features 182 are fragments ensuing from charge rearrangements by the cage. Such fragments are $\mathrm{Sc}^{+}, \mathrm{ScCN}^{+}$, ${ }_{183} \mathrm{ScC}_{2}^{+}, \mathrm{ScC}_{3}^{+}$, and so on. It is expected that if the two photon processes are initiated by the absorp184 tion of the pump and the probe pulses by the same system, then the newly formed $\mathrm{ScC}_{n}^{+}$would 185 further ionize and fragment.

186 In the present case, differences between the two delay points include a significant non-linear 187 increase of the $\mathrm{C}_{3}^{+}$intensity relative to the other small carbon species and the $\mathrm{Sc}^{+}$intensity relative 188 to the other Sc-containing fragments at the higher pulse energy, which may be indicative of 2189 photon processes. In order to obtain more evidence for this we consider the ions that are detected 

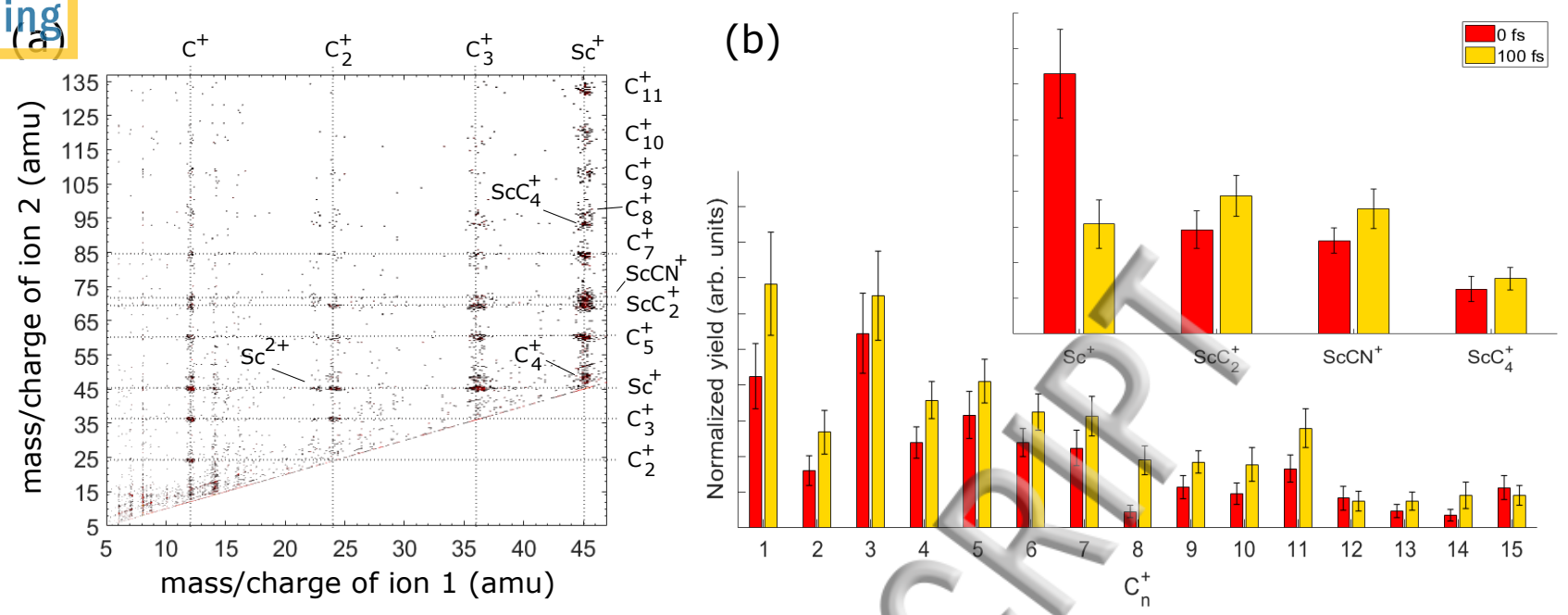

FIG. 3. (a) Photo-ion photo-ion coincidence map at the pump-probe delay of $100 \mathrm{fs}$. The mass/charge ratio is derived directly from the time-of-flight. Ions 1 and 2 are the first and second ions to hit the detector for a given FEL shot. (b) The yield corresponding to the two pump-probe delays for different carbon fragments coincident with the detection of $\mathrm{Sc}^{+}$. The inset in (b) shows the yield for species produced due to a newly formed bond between the Sc ions and fragments originating from the carbon cage. In both cases for (b), the yield is normalized by the number of FEL shots for the two pump-probe delays. Note that Fig 2 (c) and insets are plotted on the same scale as (b).

190 in coincidence.

Fig. 3 (a) shows a portion of the photo-ion photo-ion coincidence (pipico) map for ions rele192 vant to $\mathrm{Sc}^{+}$and small carbon fragment ions. Since the MCP detector count rate was $<<1 /$ shot ${ }_{193}$ (about 5\% of the FEL shot produced any hits on the MCP for the two pulses), we assume the ions 194 coincident with each other evolved from the same molecule. The prominent partner ions detected 195 in coincidence with $\mathrm{Sc}^{+}$are $\mathrm{C}_{n}^{+}$with $n$ spanning $n=1-18$. Note that it is not possible to rule out 196 the contribution of doubly or triply charged species with the same mass/charge ratio as the singly 197 charged species. However a consideration of the peak shapes in the mass spectra would indicate 198 that the contribution of multiply-charged species is very low.

199 In Fig. 3 (b), we also see a larger intensity for coincidences between $\mathrm{Sc}^{+}$and $\mathrm{C}_{n}^{+}$for the $100 \mathrm{fs}$ 200 delay data, particularly for the smallest carbon species. There is a more obvious difference in the 201 coincidences between $\mathrm{Sc}^{+}$and scandium-containing fragment ions with a very significant decrease 202 in the $\mathrm{Sc}^{+}-\mathrm{Sc}^{+}$coincidence rate for a delay of $100 \mathrm{fs}$ and increase in the $\mathrm{Sc}^{+}-\mathrm{ScC}_{2}^{+}$and $\mathrm{Sc}^{+}-$ ${ }_{203} \mathrm{ScCN}^{+}$intensities. From the pipico data, we also observe indications of $\mathrm{Sc}^{2+}$ coincident with $\mathrm{Sc}^{+}$, 
Publisłaiwgh the prominent cage fragment $\mathrm{C}_{3}^{+}$, and with ions where pieces of the cage have formed new 205 bonds with atoms originating inside the cage, i.e. $\mathrm{ScC}_{2}^{+}$and $\mathrm{ScCN}^{+}$. As there are large neutral 206 fragments from the original molecule not accounted for, these pipico structures do not form sharp 207 lines, but rather small blobs, as long as the remaining fragment(s) do not carry the majority of the 208 dissociation momentum. Somewhat surprisingly, we do not observe a noticeable amount of $\mathrm{Sc}^{2+}$ ${ }_{209}$ coincident with species other than $\mathrm{Sc}^{+}$, although we also expect these-coincidences to be difficult 210 to identify due to the higher level of multi-body fragmentation, further blurring out the ion-ion ${ }_{211}$ coincidence islands. There are also some indications of carbon fragment ions being produced in ${ }_{212}$ coincidence with other carbon fragment ions, such as $\mathrm{C}^{+}+\mathrm{C}_{2}^{+}, \mathrm{C}^{+}+\mathrm{C}_{3}^{+}, \mathrm{C}^{+}+\mathrm{C}_{4}^{+}, \mathrm{C}^{+}+\mathrm{C}_{5}^{+}, \mathrm{C}^{+}$ ${ }_{213}+\mathrm{C}_{7}^{+}$and others.

${ }_{214}$ A consideration of both the full mass spectra and the coincidence data allows us to draw ${ }_{215}$ Some interesting conclusions about the ionization and fragmentation behavior of the $\mathrm{Sc}_{3} \mathrm{~N} @ \mathrm{C}_{80}$ ${ }_{216}$ molecule. The full mass spectra, that we believe to be dominated by single-photon absorption, ${ }_{217}$ provide evidence for two distinct processes. Firstly, the strong signal from intact parent molecular ${ }_{218}$ ions with charge states from 2+ to $4+$ is indicative of Auger cascades involving predominantly the ${ }_{219}$ Sc. The doubly charged species arises from a single KLL Auger transition from the Sc (2p) with ${ }_{220}$ the emission of a high energy electron. If we consider the role of the cage which encapsulates the ${ }_{221}$ moiety, then we would see that $\mathrm{C}_{80}^{4}$, which is the charge state of the cage after charge rearrange${ }_{222}$ ment followed by removal of 2 electrons from a single $\mathrm{Sc}$, is as stable as $\mathrm{C}_{80}^{6-}$, which is the charge ${ }_{223}$ states attained by the cage in the neutral system ${ }^{39}$. This is analogous to stating that $\mathrm{Sc}_{3} \mathrm{~N} @ \mathrm{C}_{80}^{2+}$ is ${ }_{224}$ as stable as the neutral system/due to the interplay of the charge stabilization by the $\pi$ orbital and 225 the strain effect due to Coulomb repulsion. Triply and quadruply charged species most likely arise ${ }_{226}$ from LMM Auger decays within Sc. Considering that, within the cage, Sc exists on average in a ${ }_{227} 2.4+$ charge státe, no further Auger decays within the Sc system to produce higher charge states ${ }_{228}$ are possible. For the highest observable charge state, this would leave a Sc ion with an electron ${ }_{229}$ configuration of $1 s^{2} 2 s^{2} 2 p^{4} 3 s^{2} 3 p^{6}$. Any subsequent re-arrangement of electrons within the endo${ }_{230}$ hedral fullerene system to compensate for the high charge on the Sc ion would transfer electrons ${ }_{231}$ either from neighboring species inside the cage or from the carbon atoms in the cage. The lack of ${ }_{232} \mathrm{C}_{2}$ evaporation from these parent molecular ion species, such as $\mathrm{Sc}_{3} \mathrm{~N} @ \mathrm{C}_{78}, \mathrm{Sc}_{3} \mathrm{~N} @ \mathrm{C}_{76}$ and so ${ }_{233}$ on, (see Fig. 2 (a)) is a clear indication that energy transferred to internal vibrational energy of the ${ }_{234}$ cage is insufficient to produce any fragmentation on the microsecond timescale in which the ions ${ }_{235}$ reside in the extraction region of the mass spectrometer. This would be expected for sequential 
Publisłaiming sfer of electrons from the carbon cage to the Sc $(n=3)$ shell. It can therefore be assumed that ${ }_{237}$ to produce these intact parent molecular ions, the energetic electrons emitted from the KLL and ${ }_{238}$ LMM cascades do not collide inelastically with the carbon cage while exiting the molecule. If ${ }_{239}$ however, there is an inelastic collision between the 300-400 eV electrons from the LMM cascades 240 with the carbon cage, this will provide enough internal excitation to induce a phase transition in ${ }_{241}$ the hot molecule ${ }^{41}$ leading to rapid cage break-up and the characteristic mass spectrum of small ${ }_{242}$ carbon fragment ions that we observe. Extensively studied fragmentation patterns using multipho${ }_{243}$ ton laser excitation ${ }^{42}$ or collisional excitation ${ }^{43}$ transfer a wide range of energies to the fullerenes 244 and typically produce a bimodal fragment distribution. Here, we either transfer sufficient energy 245 via inelastic transfer of energy from electrons produced within the cage to cause rapid statistical ${ }_{246}$ cage break-up and the formation of singly-charged ring and chain fragments or there is insuffi${ }_{247}$ cient energy transferred to evaporate $\mathrm{C}_{2}$ from the cage on the timescale of the mass spectrometry ${ }_{248}$ detection, leaving intact parent molecular ions.
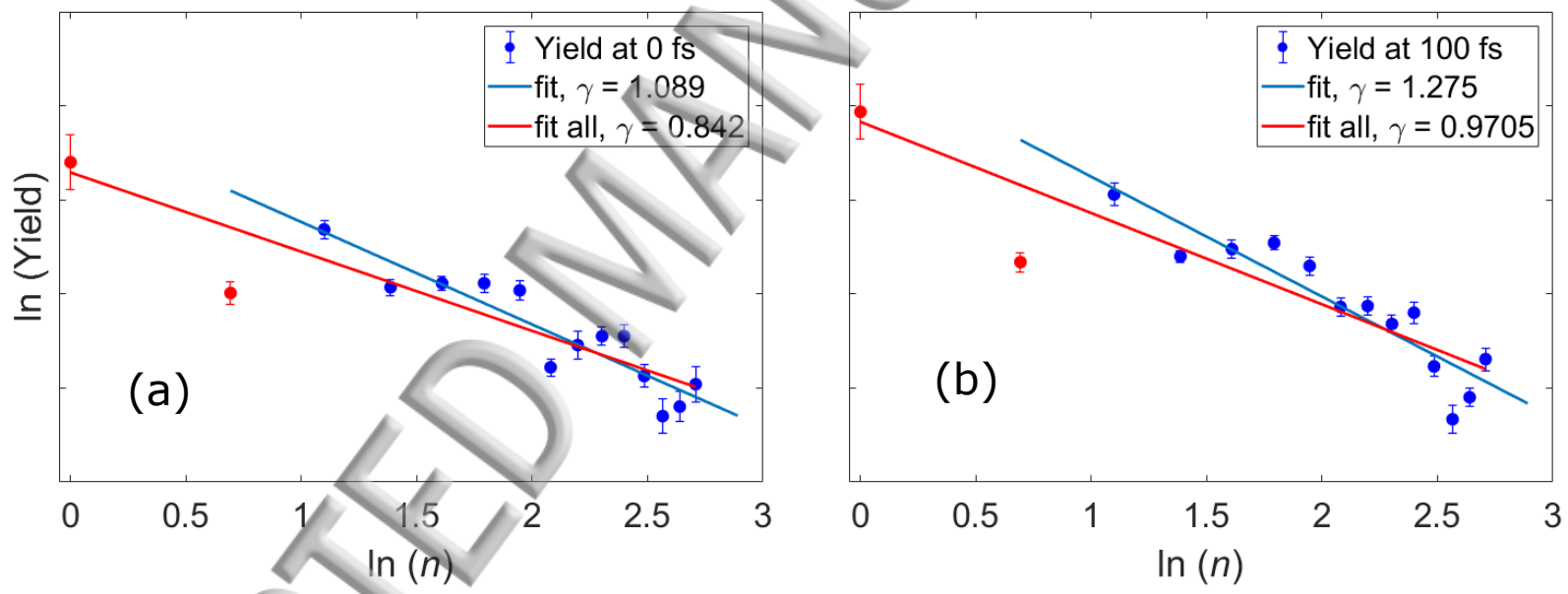

FIG. 4. Logarithmic total yields of $\mathrm{C}_{n}^{+}$for (a) 0 fs and (b) 100 fs. In both panels the blue lines show the linear fits considering fragments $\mathrm{C}_{3}^{+}$to $\mathrm{C}_{15}^{+}$, with the first two carbon fragments $\left(\mathrm{C}^{+}\right.$and $\left.\mathrm{C}_{2}^{+}\right)$excluded (shown as red dots). In (a) and (b), the red lines show the linear fits considering all the carbon fragments. Both graphs have the same scale.

249 The statistical break-up of internally excited fullerene cages had been modeled previously us250 ing both a percolation model ${ }^{44}$ and a maximum entropy model ${ }^{41}$. In the bond percolation model, 251 a power-law distribution for the small cage fragment species is predicted. The fragment ion inten${ }_{252}$ sities for both the 0 fs and 100 fs delay data have been plotted on a $\ln -\ln$ plot in Fig. 4. Figures ${ }_{253} 4$ (a) and (b) show that the data fits reasonably well to the expected power law behavior for all 
Publişalifiggments from $\mathrm{C}_{3}^{+}$to $\mathrm{C}_{15}^{+}$, as given by: $S_{n} \propto n^{-\gamma}$. Here $S_{n}$ is the yield of carbon fragments, $\mathrm{C}_{n}^{+}$, 255 and $n$ is the number of carbon atoms, while $\gamma$ is the fit parameter. The goodness of the fit, $R^{2}$, 256 for the blue fit lines for Fig. 4 (a) and (b) are 0.63, 0.82 respectively. For the fragmentation of 257 a cage system such as $\mathrm{C}_{60}$, it has been shown previously that due to finiteness and the periodic 258 nature of the system, $\gamma \approx 1.3$ is obtained for a fullerene system undergoing a phase transition by 259 emitting small carbon molecular fragments ${ }^{44}$. This value of $\gamma$ is a consequence of the amount of 260 energy transferred to the fullerene in the highly charged ion collisions ${ }^{44}$ after averaging over the 261 impact parameter dependence of the energy transfer. The gradient will change depending on how 262 much internal energy is present in the system. For very high amounts of internal energy, $\gamma$ will be 263 higher since the fragment distribution will shift more to smaller mass ions. Since, neither from the ${ }_{264}$ consideration of $\mathrm{C}^{+}$and $\mathrm{C}_{2}^{+}$nor from their omission in the overall $\ln -\mathrm{ln}$ fit, we did not obtain value 265 of $\gamma$ higher than 1.3 for any of the delays, it can be said that the measured distribution is consistent 266 with the power law behavior expected from statistical break-up as predicted by the percolation 267 model, in analogy with nuclear multi-fragmentation ${ }^{45,46}$.

268 The maximum entropy model as applied to $\mathrm{C}_{60}$ shows similar behavior but is able to reproduce ${ }_{269}$ variations in the relative intensities, including the lower than predicted yield for $\mathrm{C}^{+}$and $\mathrm{C}_{2}^{+}$by 270 taking the ionization energies and binding energies of all possible fragments into consideration ${ }^{41}$. ${ }_{271}$ For $\mathrm{C}_{60}$ the internal energy leading to the observation of the small carbon ring and chain fragment 272 ions was shown to be at about $230 \mathrm{eV}^{41}$ while for the larger endohedral system La@ $\mathrm{C}_{82}$ it was 273 predicted to be at about $300 \mathrm{eV}^{4}$. A simple extrapolation based on the number of degrees of free${ }_{274}$ dom for $\mathrm{Sc}_{3} \mathrm{~N} @ \mathrm{C}_{80}$ would lead to an expected threshold for the phase transition of about $320 \mathrm{eV}$. 275 A lower energy would be expected if the cage was multiply charged. This energy is consistent 276 with the electron energies produced by the LMM Auger transitions in Sc. The observed behavior 277 is thus supporting a model in which the cage is efficiently excited (possibly further ionized) by 278 interaction with Auger electrons as they exit the molecule, leading to a rapid energy equilibra279 tion followed by cage break-up into predominantly singly-charged fragments. Application of the ${ }_{280}$ maximum entropy model to $\mathrm{La} @ \mathrm{C}_{82}$ fragmentation was also shown to reproduce the formation of ${ }_{281} \mathrm{La} @ \mathrm{C}_{n}^{+}$fragments, similar to the scandium-containing ions observed in the current experiments ${ }^{4}$. ${ }_{282}$ The statistical nature of the break-up will also discriminate against highly charged ions in the 283 maximum entropy model due to their relatively higher ionization energies. The consideration of 284 established results from both these models provide further support for our assertion that the single 285 photon absorption process from the pump pulse alone initiates the cage breakup in the present 


\section{Publişaiłngocly.}

287 If we again turn our attention to the coincidence data in Fig. 3 (b), we see small but significant ${ }_{288}$ differences between the $0 \mathrm{fs}$ and the $100 \mathrm{fs}$ data which may be indicative of two-photon processes 289 and the timescale of intramolecular electron transfer events. The increase in the intensity of co290 incidences between $\mathrm{Sc}^{+}$and small scandium-containing fragment ions compared to coincidences 291 between two singly-charged scandium ions could be due to the dynamics of electron transfer from 292 the carbon cage to the photoionized Sc ions. At 0 fs delay, all direct or Auger induced photoion293 ization can be expected to occur on the $10 \mathrm{fs}$ timescale. Any two-photon absorption is likely to ${ }_{294}$ produce two charged Sc species within the cage giving a relatively high probability to detect a $\mathrm{Sc}^{+}$ $295-\mathrm{Sc}^{+}$coincidence signal for 0 fs delay (taking into consideration the energy and charge equili296 bration that will take place as the system is undergoing the rapid phase transition and break-up). 297 After a delay of $100 \mathrm{fs}$, there is time for electron transfer from the cage carbon atoms to the highly ${ }_{298}$ charged Sc to occur, transferring the positive charge to the cage prior to the absorption of the sec299 ond photon, and therefore giving a higher probability to detect more small charged carbon species 300 in coincidence with $\mathrm{Sc}^{+}$on the second (P)-(A) cycle. The higher yield of coincidences between ${ }_{301} \mathrm{Sc}^{+}$and scandium-containing fragment ions for $100 \mathrm{fs}$ delay may be caused by the initiation of the ${ }_{302}$ cage break-up on this timescale after the first photon absorption. In the total count of $\mathrm{Sc}^{+}$, as seen 303 in Fig. 2 (c), we do not see a significant difference between the two delays. K-shell ionization of 304 two neighboring Sc is more likely to give $\mathrm{Sc}^{+}-\mathrm{Sc}^{+}$coincidences than in the single photon case. ${ }_{305}$ We can see from Fig. $\$ 3$ that the single pulse $\mathrm{Sc}^{+}-\mathrm{Sc}^{+}$peak is much lower in intensity than the 306 pump-probe coincidence data in Fig. 3. This is reasonably convincing evidence that a majority of 307 the intensity of the $\mathrm{Sc}^{+}-\mathrm{Sc}^{+}$coincidence peak in Fig. 3 is coming from the pump-probe. At 100 $308 \mathrm{fs}$, the Fig. 3 distribution is much closer to that of Fig. S3, and we can interpret that as the holes 309 in the first ionized Sc being refilled due to transfer from the cage on a sub-100 fs timescale, so 310 that for a delay of $100 \mathrm{fs}$ the $\mathrm{Sc}$ is basically similar to that of the pristine molecule with the charge 311 transferred to the carbon cage.

\section{${ }_{312}$ IV. SUMMARY AND CONCLUSION}

313 We have experimentally probed the photo-ionization and fragmentation behavior of $\mathrm{Sc}_{3} \mathrm{~N} @ \mathrm{C}_{80}$ 314 following K-shell core ionization of the encapsulated Sc. The mass spectra provide evidence for 315 two processes leading to distinct signatures in the ion distributions: (i) multiple ionization of the 
Publisłailipgrent molecular ion via Auger cascades that transfer sufficiently little energy to vibrational exci317 tation of the molecule to survive intact during the microsecond timescale of the mass spectrometer 318 and (ii) high-energy transfer to the cage followed by cage break-up and the production of small, 319 predominantly singly-charged fragment ions. Pump-probe measurements at delays of 0 fs and 100 320 fs provided additional evidence for a "slow" (> $10 \mathrm{fs}$ ) electron transfer between the carbon cage 321 and the multiply-ionized Sc ions. Due to the "all or nothing" nature of the energy transfer to the 322 cage, the mass spectra look quite different to the typical bimodal fragment distributions that are 323 normally observed for fragmenting fullerene species. The absence of highly charged species is also 324 an unusual feature for K-shell excitation studies and is thought to be a consequence of the particu325 lar geometry of the endohedral species and the highly statistical nature of fullerene fragmentation. ${ }_{326}$ Although the current data is of a rather preliminary nature and requires further experimental and 327 theoretical study to fully unravel the complex dynamics of the studied system, we have shown ${ }_{328}$ the feasibility of $\mathrm{x}$-ray FEL pump-probe experiments to probe the complex electron and nuclear 329 dynamics of large molecular systems.

\section{${ }_{330}$ SUPPLEMENTARY MATERIAL}

${ }_{331}$ See 'Supplementary Information' for the figures S1, S2, S3, S4 and S5 mentioned in the 332 manuscript.

\section{ACKNOWLEDGEMENT}

${ }_{334}$ This work was funded by the Chemical Sciences, Geosciences, and Biosciences Division, ${ }_{335}$ Office of Basic Energy Sciences, Office of Science, U.S. Department of Energy, grant No. ${ }_{336}$ DE-SC0012376. T. T. gratefully acknowledges support by the JSPS KAKENHI Grant Number ${ }_{337}$ JP16J02270. H. F., K. N., and K. U. were supported by "Dynamic Alliance for Open Innovation ${ }_{338}$ Bridging Human, Environment and Materials" from the Ministry of Education, Culture, Sports, ${ }_{339}$ Science and Technology of Japan (MEXT), by the Research Program of "Dynamic Alliance 340 for Open Innovation Bridging Human, Environment and Materials" in "Network Joint Research ${ }_{341}$ Center for Materials and Devices", by the Japan Society for the Promotion of Science (JSPS) ${ }_{342}$ KAKENHI Grant Numbers JP15K17487, and by the IMRAM project. D.Y. was supported by a ${ }_{343}$ Grant-in-Aid of Tohoku University Institute for Promoting Graduate Degree Programs Division 
Publishaifog Interdisciplinary Advanced Research and Education.

${ }_{345}$ We would like to thank the SACLA Accelerator scientists and beamline staff for the technical 346 and experimental help provided during the beamtime.

\section{REFERENCES}

${ }^{1}$ H. Shinohara and N. Tagmatarchis, Endohedral Metallofullerenes: Fullerenes with Metal Inside (Wiley, 2015).

${ }^{2}$ A. Rodríguez-Fortea, A. L. Balch, and J. M. Poblet, "Endohedral metallofullerenes: a unique host-guest association," Chemical Society Reviews 40, 3551-3563 (2011).

${ }^{3}$ Y. Wang, R. Yamachika, A. Wachowiak, M. Grobis, and M. F. Crommie, "Tuning fulleride electronic structure and molecular ordering via variable layer index," Nature Materials 7, 194 (2008).

${ }^{4}$ A. Lassesson, A. Gromov, K. Mehlig, A. Taninaka, H. Shinohara, and E. E. B. Campbell, "Formation of small lanthanum-carbide ions from laser induced fragmentation of $\mathrm{La}_{2} @ \mathrm{C}_{82}$," The Journal of Chemical Physics 119, 5591-5600 (2003).

${ }^{5}$ H. Xiong, L. Fang, T. Osipov, N. G. Kling, T. J. A. Wolf, E. Sistrunk, R. Obaid, M. Gühr, and N. Berrah, "Fragmentation of endohedral fullerene $\mathrm{Ho}_{3} \mathrm{~N} @ \mathrm{C}_{80}$ in an intense femtosecond near-infrared laser field," Physical Review A 97, 023419 (2018).

${ }^{6}$ H. Xiong, R. Obaid, L. Fang, C. Bomme, N. G. Kling, U. Ablikim, V. Petrovic, C. E. LiekhusSchmaltz, H. Li, R. C. Bilodéau, et al., "Soft x-ray induced ionization and fragmentation dynamics of $\mathrm{Sc}_{3} \mathrm{~N} @ \mathrm{C}_{80}$ investigated using an ion-ion-coincidence momentum-imaging technique," Physical Review A 96, 033408 (2017).

${ }^{7}$ J. Hellhund, A. Borovik Jr, K. Holste, S. Klumpp, M. Martins, S. Ricz, S. Schippers, and A. Mueller, "Photoionization and photofragmentation of multiply charged $\mathrm{Lu}_{3} \mathrm{~N} @ \mathrm{C}_{80}$ ions," Physical Review A 92, 013413 (2015).

${ }^{8}$ A. Müller, S. Schippers, R. A. Phaneuf, M. Habibi, D. Esteves, J. C. Wang, A. L. D. Kilcoyne, A. Aguilar, S. Yang, and L. Dunsch, "Photoionization of the endohedral fullerene ions $\mathrm{Sc}_{3} \mathrm{~N} @ \mathrm{C}_{80}$ and $\mathrm{Ce} @ \mathrm{C}_{82}$ by synchrotron radiation," Journal of Physics: Conference Series 88, 012038 (2007).

${ }^{9}$ A. Müller, M. Martins, A. Kilcoyne, R. Phaneuf, J. Hellhund, A. Borovik Jr, K. Holste, S. Bari, T. Buhr, S. Klumpp, et al., "Photoionization and photofragmentation of singly charged positive 
Publisłaingnd negative $\mathrm{Sc}_{3} \mathrm{~N} @ \mathrm{C}_{80}$ endohedral fullerene ions," Physical Review A 99, 063401 (2019).

${ }_{375}{ }^{10} \mathrm{~B}$. Mignolet, T. Kus, and F. Remacle, "Imaging orbitals by ionization or electron attachment:

376 The role of dyson orbitals," in Imaging and Manipulating Molecular Orbitals (Springer, 2013) 377 pp. 41-54.

${ }^{378}{ }^{11}$ N. Berrah, J. Bozek, J. Costello, S. Düsterer, L. Fang, J. Feldhaus, H. Fukuzawa, M. Hoener, 379 Y. Jiang, P. Johnsson, et al., "Non-linear processes in the interaction of atoms and molecules ${ }_{380}$ with intense EUV and x-ray fields from SASE free electron lasers (FELs)," Journal of Modern 381 Optics 57, 1015-1040 (2010).

${ }_{382}{ }^{12}$ A. Rudenko, L. Inhester, K. Hanasaki, X. Li, S. Robatjazi, B. Erk, R. Boll, K. Toyota, Y. Hao, 383 O. Vendrell, et al., "Femtosecond response of polyatomic molecules to ultra-intense hard x384 rays," Nature 546, 129 (2017).

${ }_{385}^{13}$ L. Young, E. Kanter, B. Krässig, Y. Li, A. March, S. Pratt, R. Santra, S. Southworth, ${ }_{386}$ N. Rohringer, L. DiMauro, et al., "Femtosecond electronic response of atoms to ultra-intense 387 X-rays," Nature 466, 56 (2010).

${ }_{388}^{14}$ L. Fang, M. Hoener, O. Gessner, F. Tarantelli, S. T. Pratt, O. Kornilov, C. Buth, M. Gühr, ${ }_{389}$ E. P. Kanter, C. Bostedt, et al., "Double core-hole production in $\mathrm{N}_{2}$ : beating the Auger clock," 390 Physical Review Letters 105, 083005 (2010).

${ }_{391}{ }^{15}$ M. Hoener, L. Fang, O. Kornilov, O. Gessner, S. T. Pratt, M. Gühr, E. P. Kanter, C. Blaga, 392 C. Bostedt, J. D. Bozek, et al., "Ultraintense x-ray induced ionization, dissociation, and frus${ }_{394}^{16}$ B. Murphy, T. Osipov, Z. Jurek, L. Fang, S.-K. Son, M. Mucke, J. Eland, V. Zhaunerchyk, 395 R. Feifel, L. Avaldi, et al., "Femtosecond x-ray-induced explosion of $\mathrm{C}_{60}$ at extreme intensity," ${ }_{396}$ Nature Communications 5, 4281 (2014).

${ }_{397}^{17}$ B. Abbey, R. A. Dilanian, C. Darmanin, R. A. Ryan, C. T. Putkunz, A. V. Martin, D. Wood, 398 V. Streltsov, M. W. M. Jones, N. Gaffney, F. Hofmann, G. J. Williams, S. Boutet, M. Messer${ }_{399}$ schmidt, M. M. Seibert, S. Williams, E. Curwood, E. Balaur, A. G. Peele, K. A. Nugent, 400 and H. M. Quiney, "X-ray laser-induced electron dynamics observed by femtosecond diffrac401 tion from nanocrystals of Buckminsterfullerene," Science Advances 2 (2016), 10.1126/sci${ }_{402}$ adv.1601186.

${ }_{403}^{18}$ M. O. Krause, “Atomic radiative and radiationless yields for K and L shells," Journal of Physical 404 and Chemical Reference Data 8, 307-327 (1979).

${ }_{405}{ }^{19}$ C. E. Moore and H. N. Russell, "Binding energies for electrons of different types," Journal of 
Publisłaingesearch of the National Bureau of Standards 48, 2285 (1952).

${ }_{407}^{20}$ B. Dünser, M. Lezius, P. Scheier, H. Deutsch, and T. Märk, "Electron impact ionization of C 60 ," 408 Physical Review Letters 74, 3364 (1995).

${ }_{409}^{21}$ A. A. Vostrikov, D. Y. Dubnov, and A. A. Agarkov, "Inelastic interaction of an electron with a ${ }_{410} \quad \mathrm{C}_{60}$ cluster," High Temperature 39, 22-30 (2001).

${ }_{411}^{22}$ M. Yabashi, H. Tanaka, and T. Ishikawa, "Overview of the SACLA facility," Journal of Syn412 chrotron Radiation 22, 477-484 (2015).

${ }_{413}^{23}$ T. Ishikawa, H. Aoyagi, T. Asaka, Y. Asano, N. Azumi, T. Bizen, H. Ego, K. Fukami, T. Fukui, ${ }_{414}$ Y. Furukawa, et al., "A compact x-ray free-electron laser emitting in the sub-ångström region," 415 Nature Photonics 6, 540 (2012).

${ }_{416}^{24}$ H. Fukuzawa, K. Nagaya, and K. Ueda, "Advances in instrumentation for gas-phase spec${ }_{417}$ troscopy and diffraction with short-wavelength free electron lasers," Nuclear Instruments and ${ }_{418}$ Methods in Physics Research Section A: Accelerators, Spectrometers, Detectors and Associated ${ }_{419}$ Equipment 907, 116-131 (2018).

${ }_{420}{ }^{25}$ T. Hara, Y. Inubushi, T. Katayama, T. Sato, H. Tanaka, T. Tanaka, T. Togashi, K. Togawa, ${ }_{421}$ K. Tono, M. Yabashi, et al., "Two-colour hard x-ray free-electron laser with wide tunability," 422 Nature Communications 4, 2919 (2013)

${ }_{423}{ }^{26} \mathrm{~J}$. A. Bearden and A. Burr, "Reevaluation of X-ray atomic energy levels," Reviews of Modern ${ }_{424}$ Physics 39, 125 (1967).

${ }_{425}{ }^{27}$ K. Tamasaku, Y. Inubushi, I. Inoue, K. Tono, M. Yabashi, and T. Ishikawa, "Inline spectrometer ${ }_{426}$ for shot-by-shot determination of pulse energies of a two-color x-ray free-electron laser,' Journal 427 of Synchrotron Radiation 23, 331-333 (2016).

${ }_{428}^{28}$ H. Yumoto, H. Mimura, S. Matsuyama, T. Koyama, Y. Hachisu, T. Kimura, H. Yokoyama, ${ }_{429}$ J. Kim, Y. Sano, K. Tono, et al., "Micro-focusing of hard X-ray free electron laser radiation ${ }_{430}$ using Kirkpatrick-Baez mirror system," in Journal of Physics: Conference Series, Vol. 425 (IOP 431 Publishing, 2013) p. 052022.

${ }_{432}^{29}$ Y. Inubushi, K. Tono, T. Togashi, T. Sato, T. Hatsui, T. Kameshima, K. Togawa, T. Hara, T. Tanaka, H. Tanaka, et al., "Determination of the pulse duration of an X-ray free electron ${ }_{434}$ laser using highly resolved single-shot spectra," Physical Review Letters 109, 144801 (2012). ${ }^{435}{ }^{30}$ K. Motomura, L. Foucar, A. Czasch, N. Saito, O. Jagutzki, H. Schmidt-Böcking, R. Dörner, X.J. Liu, H. Fukuzawa, G. Prümper, et al., "Multi-coincidence ion detection system for EUV-FEL ${ }_{437}$ fragmentation experiments at SPring-8," Nuclear Instruments and Methods in Physics Research 
Publisłaingection A: Accelerators, Spectrometers, Detectors and Associated Equipment 606, 770-773 439 (2009).

${ }_{440}^{31}$ B. Rudek, K. Toyota, L. Foucar, B. Erk, R. Boll, C. Bomme, J. Correa, S. Carron, S. Boutet, ${ }_{441}$ G. J. Williams, et al., "Relativistic and resonant effects in the ionization of heavy atoms by ${ }_{442}$ ultra-intense hard X-rays," Nature Communications 9, 4200 (2018).

${ }_{443}^{32}$ N. Berrah, B. Murphy, H. Xiong, L. Fang, T. Osipov, E. Kukk, M. Guehr, R. Feifel, V. Petro${ }_{444}$ vic, K. Ferguson, et al., "Femtosecond x-ray-induced fragmentation of fullerenes," Journal of 445 Modern Optics 63, 390-401 (2016).

${ }_{446}^{33}$ R. Obaid, C. Buth, G. L. Dakovski, R. Beerwerth, M. Holmes, J. Aldrich, M.-F. Lin, M. Minitti, ${ }_{447}$ T. Osipov, W. Schlotter, et al., "LCLS in-photon out: fluorescence measurement of neon using 448 soft X-rays," Journal of Physics B: Atomic, Molecular and Optical Physics 51, 034003 (2018).

${ }_{449}{ }^{34}$ T. Ekeberg, M. Svenda, C. Abergel, F. R. Maia, V. Seltzer, J.-M. Claverie, M. Hantke, O. Jöns${ }_{450}$ son, C. Nettelblad, G. Van Der Schot, et al., "Three-dimensional reconstruction of the giant 451 mimivirus particle with an x-ray free-electron laser," Physical Review Letters 114, 098102 $452 \quad(2015)$.

${ }_{453}{ }^{35}$ C. Bostedt, S. Boutet, D. M. Fritz, Z. Huang, H. J. Lee, H. T. Lemke, A. Robert, W. F. Schlotter, ${ }_{454}$ J. J. Turner, and G. J. Williams, "Linac Coherent Light Source: The first five years," Reviews 455 of Modern Physics 88, 015007 (2016).

${ }_{456}{ }^{36} \mathrm{~N}$. Berrah, "A perspective for investigating photo-induced molecular dynamics from within ${ }_{457}$ with femtosecond free electron lasers,” Physical Chemistry Chemical Physics 19, 19536-19544 458 (2017).

${ }_{459}^{37}$ L. Young, K. Ueda, M. Gühr, P. H. Bucksbaum, M. Simon, S. Mukamel, N. Rohringer, K. C. 460 Prince, C. Masciovecchio, M. Meyer, et al., "Roadmap of ultrafast X-ray atomic and molecular ${ }_{461}$ physics," Journal of Physics B: Atomic, Molecular and Optical Physics 51, 032003 (2018).

${ }_{462}^{38}$ L. Alvarez, T. Pichler, P. Georgi, T. Schwieger, H. Peisert, L. Dunsch, Z. Hu, M. Knupfer, ${ }_{463}$ J. Fink, P. Bressler, M. Mast, and M. S. Golden, "Electronic structure of pristine and intercalated ${ }_{464} \mathrm{Sc}_{3} \mathrm{~N} @ \mathrm{C}_{80}$ metallofullerene,” Phys. Rev. B 66, 035107 (2002).

${ }_{465}{ }^{39}$ Y. Wang, S. Díaz-Tendero, M. Alcamí, and F. Martín, "Cage connectivity and frontier $\pi$ orbitals 466 govern the relative stability of charged fullerene isomers," Nature Chemistry 7, 927 (2015).

${ }_{467}^{40}$ J. Hoszowska, A. Kheifets, J.-C. Dousse, M. Berset, I. Bray, W. Cao, K. Fennane, Y. Kayser, M. Kavčič, J. Szlachetko, et al., "Physical mechanisms and scaling laws of K-shell double photoionization,” Physical Review Letters 102, 073006 (2009). 


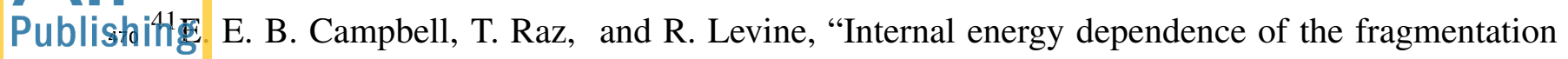
${ }_{471}$ patterns of $\mathrm{C}_{60}$ and $\mathrm{C}_{60}^{+}$," Chemical Physics Letters 253, 261-267 (1996).

${ }_{472}^{42}$ H. Hohmann, R. Ehlich, S. Furrer, O. Kittelmann, J. Ringling, and E. E. B. Campbell, 473 “Photofragmentation of $\mathrm{C}_{60}$," Zeitschrift für Physik D Atoms, Molecules and Clusters 33, 143$474 \quad 151(1995)$.

${ }_{475}^{43}$ Z. Jurek, B. Ziaja, and R. Santra, "Applicability of the classical molecular dynamics method to 476 study X-ray irradiated molecular systems," Journal of Physics B: Atomic, Molecular and Optical 477 Physics 47, 124036 (2014).

${ }_{478}^{44}$ S. Cheng, H. Berry, R. Dunford, H. Esbensen, D. Gemmell, E. Kanter, T. LeBrun, and W. Bauer, 479 "Ionization and fragmentation of $\mathrm{C}_{60}$ by highly charged, high-energy xenon ions," Physical Re$480 \quad$ view A 54, 3182 (1996).

${ }_{481}^{45}$ D. Gruyer, J. Frankland, R. Botet, M. Płoszajczak, E. Bonnet, A. Chbihi, G. Ademard, ${ }_{482}$ M. Boisjoli, B. Borderie, R. Bougault, et al., "Nuclear multifragmentation time scale and fluc483 tuations of the largest fragment size," Physical Review Letters 110, 172701 (2013).

${ }^{484}{ }^{46}$ A. Hirsch, A. Bujak, J. Finn, L. Gutay, R. Minich, N. Porile, R. Scharenberg, B. Stringfellow, and F. Turkot, "Experimental results from high energy proton-nucleus interactions, critical phenomena, and the thermal liquid drop model of fragment production," Physical Review C 29, 508 (1984). 

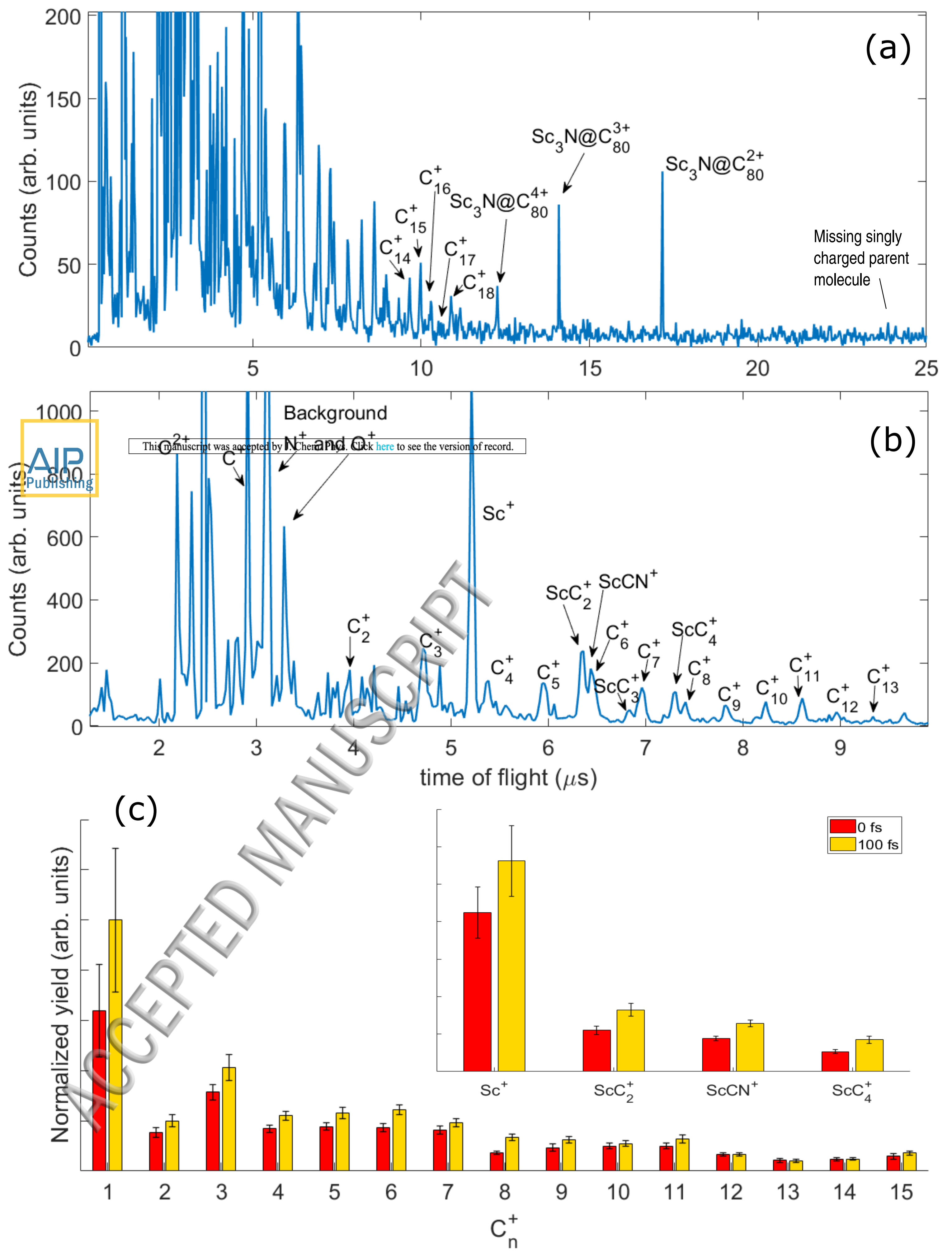
(a)

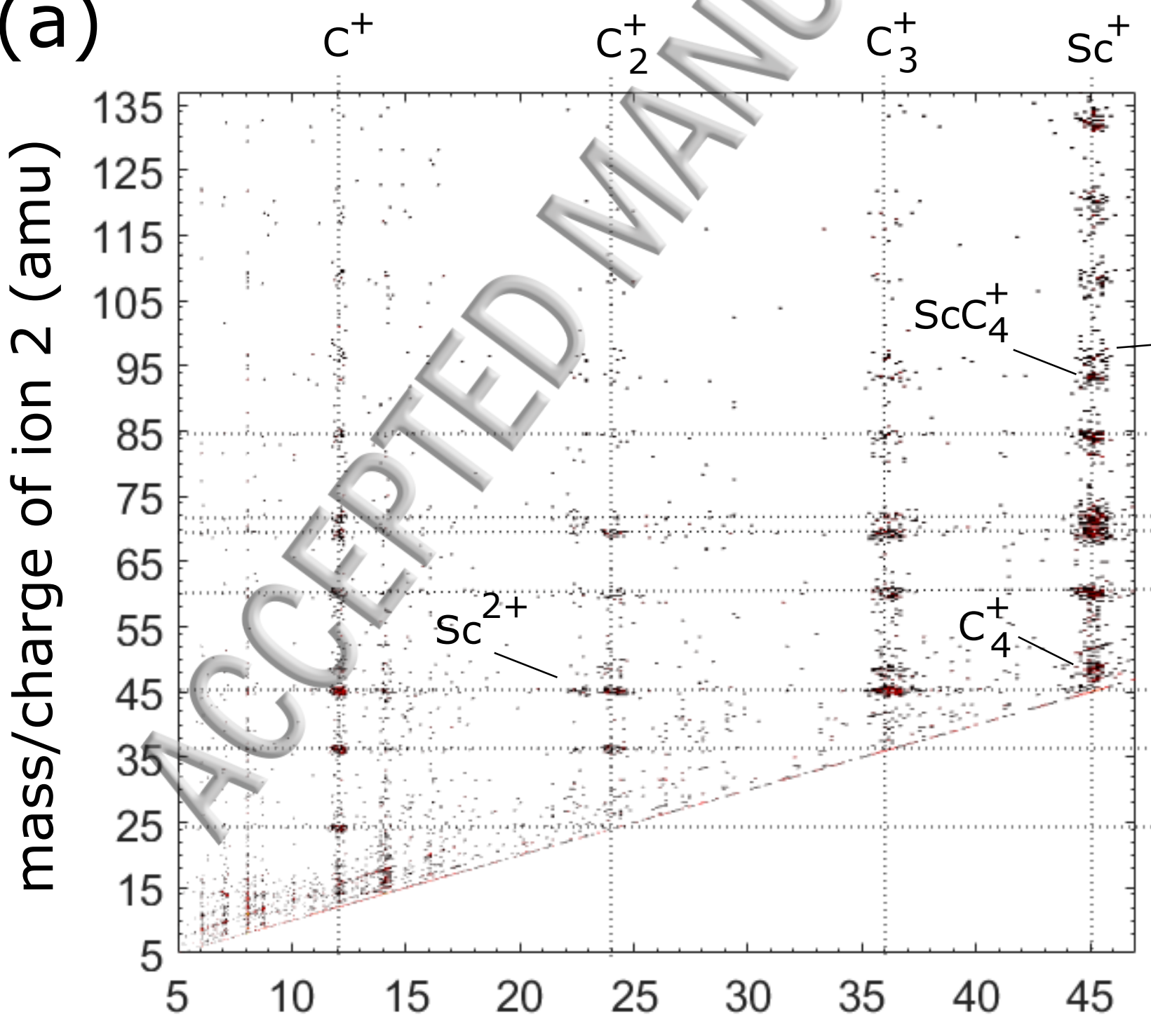
mass/charge of ion 1 (amu)

(b)

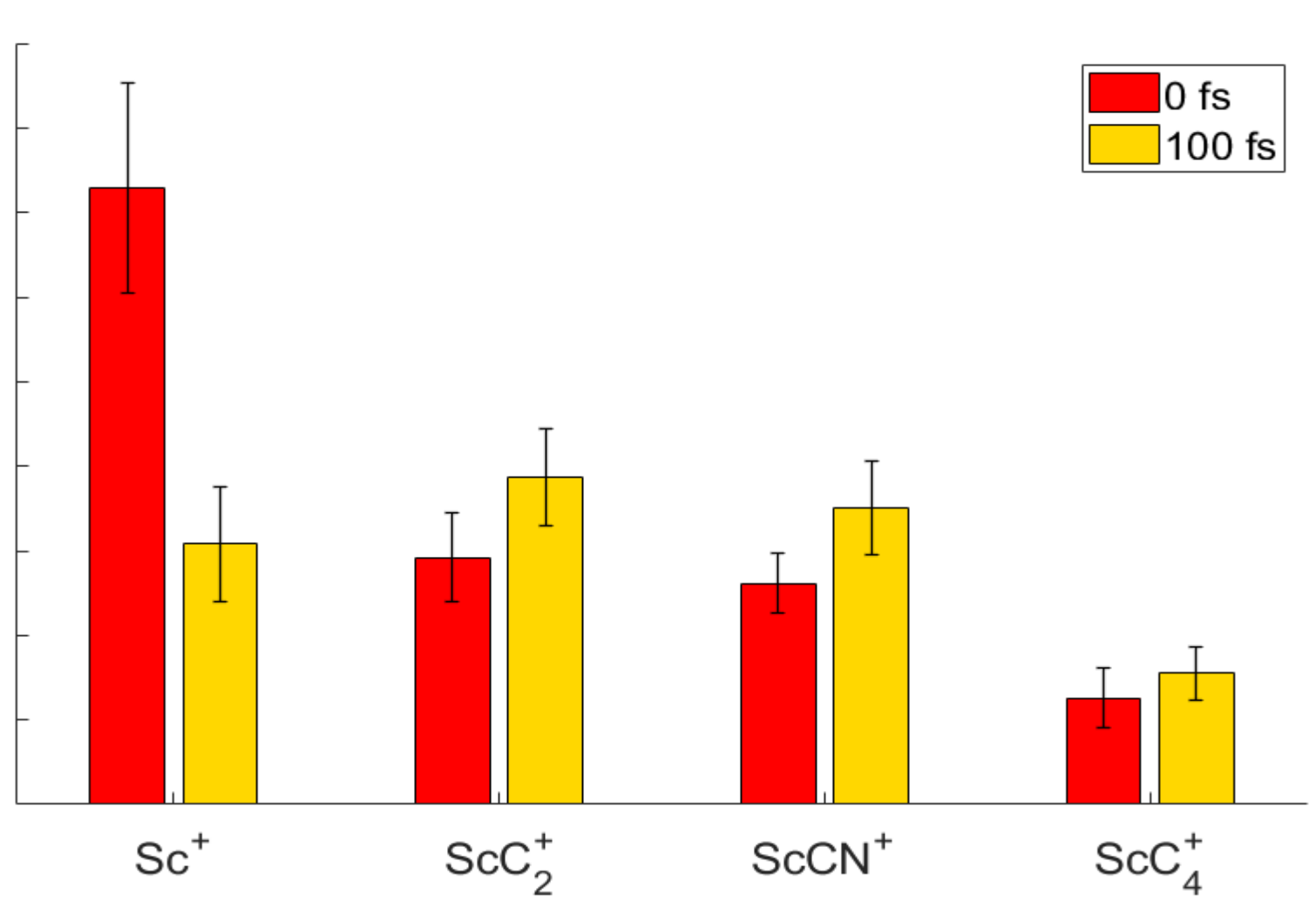

$\mathrm{Sc}^{+}$
$\mathrm{C}_{3}^{+}$

$\mathrm{C}_{2}^{+}$ 


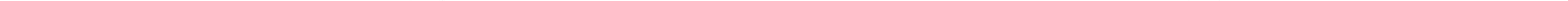

